\title{
Pap Testing in the USA and Potential Association with HPV Vaccination: a Cross-sectional Analysis of the BRFSS Data (2007-2016)
}

\author{
Dongyu Zhang, $P h D^{7}$ and Xuezheng Sun, $P h D^{2}$ \\ 'Department of Oncology, Georgetown University School of Medicine, Washington, DC, USA; ${ }^{2}$ Department of Epidemiology, University of North \\ Carolina at Chapel Hill Gillings School of Global Public Health, Chapel Hill, NC, USA.
}

KEY WORDS: cancer screening; Pap test; cervical cancer prevention; HPV vaccination.

$\mathrm{J}$ Gen Intern Med 34(1):17-9

DOI: $10.1007 / \mathrm{s} 11606-018-4652-\mathrm{y}$

(c) Society of General Internal Medicine 2018

\section{INTRODUCTION}

The US Preventive Services Task Force (USPSTF) recommends women aged 21-65 years should undergo Papanicolaou test (Pap test) every 3 years to prevent cervical cancer. ${ }^{1}$ However, no nationwide representative populationbased research described Pap test use from a temporal perspective in the past decade. This brief report describes the temporal trend of Pap test use in the USA between 2007 and 2016. Considering that human papillomavirus (HPV) vaccination may affect screening behaviors, we further assessed the 10-year trend of HPV vaccination and its association with Pap test use.

\section{METHODS}

We used the Behavioral Risk Factor Surveillance System (BRFSS) data for the current analysis, and five intervals were generated (2007-2008, 2009-2010, 2011-2012, 2013-2014, and 2015-2016).

First, we summarized the trend of Pap test within the 10year period by treating women having a test within the last 3 years as the outcome; the analysis was conducted for the overall sample and subgroups defined by age and race. We further investigated the trend by treating Pap test within the last 5 years as the outcome to examine if screening behaviors shifted to a long-interval pattern. Generalized linear regression was used to calculate the average rate difference (ARD) per each interval of Pap test. HPV vaccination trend was examined using the same rationale. Multivariable logistic regressions were used to investigate the association between HPV vaccination and Pap test in the last 3 years. All statistical analyses were corrected for sampling weight and were conducted using Stata 13.0 (College Station, TX, StataCorp, LLP).

Published online September 11, 2018

\section{RESULTS}

A total of $1,433,358$ women were included in the present analysis. Overall, over half of the participants $(n=762,836$; weighted percentage, $71.8 \%$ ) were younger than 60 years or white women $(n=1,180,138$; weighted percentage, $77.7 \%)$. During the 10 -year period, $72.5 \%$ and $78.0 \%$ of women reported having a Pap test within the last 3 and 5 years, respectively. A monotonously decreased pattern of Pap test was observed among the overall population and subgroups defined by age or race (Table 1). The HPV vaccination rate increased across the 10-year period, with an ARD of 3.6\% $(95 \% \mathrm{CI}=3.2 \%, 4.1 \%)$ per each interval. A significant inverse association between HPV vaccination and Pap test within 3 years was observed among white women $(\mathrm{OR}=0.82,95 \%$ $\mathrm{CI}=0.67,0.99)$. The association patterns did not change in women without a history of hysterectomy (Table 2).

\section{DISCUSSION}

A previous study reported a steady Pap test rate between 1994 and $2004,{ }^{2}$ whereas we observed a declining trend between 2007 and 2016, and the pattern is consistent by age and race. There is no evidence suggesting a shift to long-interval Pap test pattern ( 3 to 5 years). Among white women, the decreased use of Pap test may be partially explained by the increasing HPV vaccination.

The inverse association between HPV vaccination and cervical cancer screening among white women suggests a heterogeneous association pattern across different races; this also raised the need for a more targeted health promotion program to enhance cervical cancer screening and reduce disease burden. Given that Pap test and HPV vaccination had different temporal patterns within 2007-2016, there could be a tendency that the emphasis of cervical cancer prevention shifted from secondary to primary prevention in the USA.

The Healthy People 2020 Program, which was established by the US Department of Health and Human Services and public stakeholders, aims to increase Pap test use in the USA. ${ }^{3,}{ }^{4}$ However, our outcomes suggest this target has not yet been accomplished. Although the recent incidence and mortality of cervical cancer are decreasing slightly annually, ${ }^{5}$ 
Table 1 Overall and Age/Race-Categorized Trend of Pap Test Use and HPV Vaccination

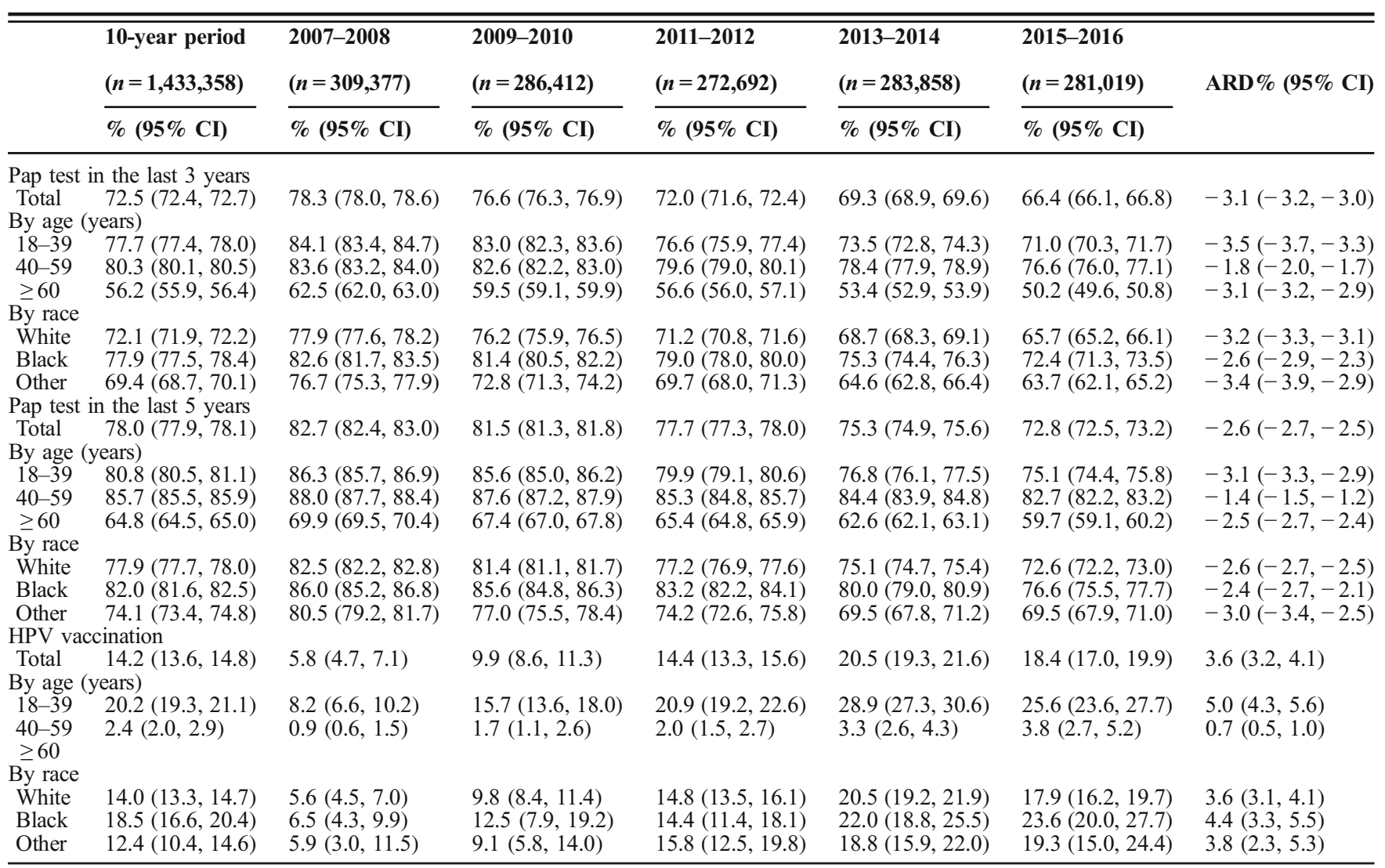

Pap test Papanicolaou test, HPV human papillomavirus, ARD average rate difference by each interval, CI confidence interval No estimates of HPV vaccination were reported for women $\geq 60$ years because of sparse data

We reported point estimates of weighted rates and 95\% confidence intervals of Pap test use and history of HPV vaccination

the long-term impact of the decline in Pap test is not clear. Future research should monitor whether this has a negative impact on cervical cancer incidence and mortality.

On the other hand, a simulation study ${ }^{6}$ reported vaccinated women could start screening at later ages and undergo Pap test less frequently to obtain more health benefit and reduce po- tential harm. Since HPV vaccination can significantly reduce the lifetime risk of cervical cancer, health practitioners should consider whether it is necessary to lengthen screening intervals for vaccinated women.

Last, our results need to be interpreted with cautions that information about screening and vaccination was obtained by

Table 2 Associations Between HPV Vaccination and Pap Test Use in the Last 3 years Among the Overall Sample and Subgroups Defined by Age/Race

\begin{tabular}{llccc}
\hline \hline & COR and 95\% CI & aOR and 95\% CI* & aOR and 95\% CI & aOR and 95\% CI* \\
\hline $\begin{array}{l}\text { HPV vaccination } \\
\text { Total }\end{array}$ & $0.69(0.61,0.78)$ & $0.77(0.68,0.87)$ & $0.89(0.76,1.05)$ & $0.88(0.74,1.04)$ \\
$\begin{array}{l}\text { By age (years) } \\
\text { 18-39 }\end{array}$ & $0.73(0.64,0.83)$ & $0.83(0.72,0.95)$ & $1.02(0.86,1.21)$ & $0.99(0.83,1.19)$ \\
40-59 & $1.27(0.85,1.90)$ & $1.36(0.90,2.06)$ & $1.31(0.87,1.98)$ & $1.25(0.77,2.03)$ \\
$\begin{array}{l}\text { B } 60 \\
\text { By race }\end{array}$ & & & & \\
White & $0.62(0.55,0.71)$ & $0.69(0.60,0.79)$ & $0.82(0.67,0.99)$ & $0.81(0.66,1.00)$ \\
Black & $0.72(0.50,1.04)$ & $0.81(0.56,1.16)$ & $1.05(0.68,1.61)$ & $1.01(0.65,1.58)$ \\
Other & $0.87(0.60,1.25)$ & $1.05(0.75,1.47)$ & $1.11(0.73,1.70)$ & $1.03(0.68,1.58)$ \\
\hline
\end{tabular}

HPV human papillomavirus, cOR crude odds ratio, aOR adjusted odds ratio, CI confidence interval

Women who never had HPV vaccination were treated as the reference group analysis

No effect measures were reported for women $\geq 60$ years because of sparse data

*The model adjusted for survey year

${ }^{\dagger}$ The model adjusted for age, race, income, education, marriage status, body mass index, smoking status, cardiometabolic disease, health coverage, clinical checkup, and survey year

${ }^{\ddagger}$ The model restricted to women without hysterectomy history and adjusted for age, race, income, education, marriage status, body mass index, smoking status, cardiometabolic disease, health coverage, clinical checkup, and survey year 
self-report, which can be less valid compared to medical record review. Moreover, the cross-sectional design can bring difficulties to causal interpretation.

Corresponding Author: Dongyu Zhang, PhD; Department of OncologyGeorgetown University School of Medicine, Washington, DC, USA (e-mail: dz216@georgetown.edu).

\section{Compliance with Ethical Standards:}

Conflict of Interest: The authors declare that they do not have a conflict of interest.

Ethical Approval: IRB approval was not required as our study used publicly available dataset.

\section{REFERENCES}

1. Cervical cancer: screening. https://www.uspreventiveservicestaskforce. org/Page/Document/UpdateSummaryFinal/cervical-cancer-screening. Last accessed 7 May 2018.

2. Doescher MP, Jackson JE. Trends in cervical and breast cancer screening practices among women in rural and urban areas of the United States. J Public Health Manag Pract 2009; 15(3):200-209.

3. Healthy people 2020: increase the proportion of women who receive a cervical cancer screening based on the most recent guidelines. https:// www.healthypeople.gov/2020/data-search/Search-the-Data\# objid=4053. Last accessed 8 May 2018.

4. Introducing healthy people 2020. https://www.healthypeople.gov/2020/ About-Healthy-People. Last accessed 8 May 2018.

5. Cervical Cancer Trends. https://www.cdc.gov/cancer/cervical/statistics/ trends.htm. Last accessed 31 May 2018.

6. Kim JJ, Burger EA, Sy S, Campos NG. Optimal cervical cancer screening in women vaccinated against human papillomavirus. J Natl Cancer Inst 2017;109(2). https://doi.org/10.1093/jnci/djw216 